\title{
Application of least square curve fitting algorithm based on LabVIEW in pressure detection system
}

\author{
Wei SUN ${ }^{1,}$, Feng ZUO ${ }^{1, b}$, Aihua DONG ${ }^{1, c}$, Liya ZHOU ${ }^{2, d}$ \\ ${ }^{1}$ College of Information Science and Technology, Dong Hua University, Shanghai, China \\ ${ }^{2}$ College of Textile, Dong Hua University, Shanghai, China

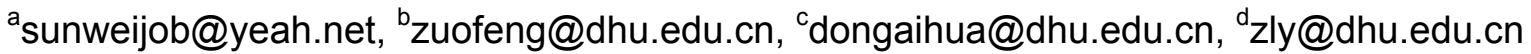

Keywords: LabVIEW; Data Acquisition; Flexiforce; Least Square

\begin{abstract}
This paper introduces the constitution and working principle of a LabVIEW platform-based pressure detection system. Flexiforce transducer is engaged in the detection system for data acquisition of clothing pressure. Particularly, least square curve fitting algorithm principle and linear regression analysis technique are expounded in the system. Therefore, the proposed pressure detection system could analyze real-time data of pressure and conduct objective test dynamically. This scheme is not only suitable for the design of pressure detection system, but also has certain guiding significance for a wide range of sensor detection system design.
\end{abstract}

\section{Introduction}

The research of clothing pressure mainly focused on establishment of theoretical model and continuous improvement of the detection method ${ }^{[1]}$. Typical clothing pressure detection methods include mechanical, pneumatic and pressure sensor measurement.

Since the output of the pressure sensor is an analog voltage signal, it is necessary to adopt curve fitting technique to implement system calibration. The objective of the system calibration is to determine input - output relationship, eliminate the system error, improve the performance of the system, and achieve signal conversion from voltage to the pressure. Curve fitting technique is a kind of data processing method, which is used to describe the function relationship between the discrete points in the plane and the discrete points on the plane ${ }^{[2]}$. Least squares fitting algorithm is practical engineering applications fitting method. The principle of the algorithm is to obtain the most reliable measurements to eliminate the sum of squared residuals of measurement results ${ }^{[3]}$.

At the same time, in recent years, the rapid development of the virtual instrument LabVIEW technology using graphical G programming language improves the efficiency of programming and significantly speeds up the development process of automated testing technology.

To overcome the shortcomings of traditional clothing pressure detection system, for instance, lacking of dynamic test, this paper develops a pressure detection system with 8 - channel signal acquisition function. In this pressure detection system, LabVIEW is employed as the development platform and pressure-sensitive semiconductor thin film pressure sensors named Flexiforce from American Tekscan Company are engaged for pressure measurement. Combining with least square fitting algorithm, the proposed system is successfully applied in dynamic clothing pressure test.

\section{Components and Principle of Detecting System}

Based on the LabVIEW development platform, pressure detection system consists of Flexiforce sensor, MC34074 operational amplifier circuit, NI SCXI signal conditioning data acquisition board and the computer terminal. The work principle diagram is shown in Fig. 1. 


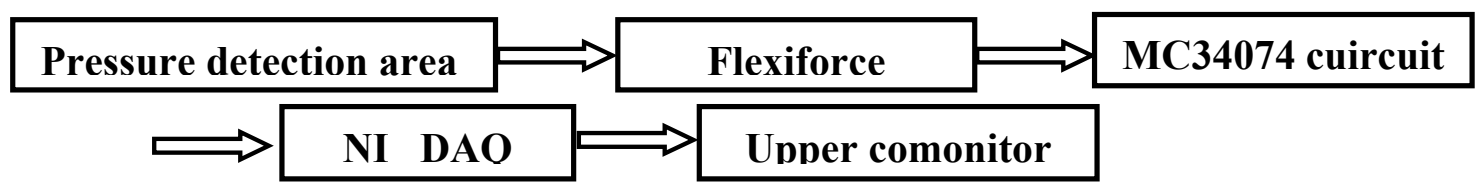

Fig. 1 Principle diagram of pressure detection system

The front end of the system is designed with ultra-thin Flexiforce force sensor, which can be used to detect the pressure of the measuring point. The main form of the traditional pressure sensor is the strain type, capacitance type, voltage type and resistance type, and so on. Compared with the traditional pressure sensor, Flexiforce sensor has the advantages of obvious characteristics, such as paper - like light, flexible, and durability, so it is suitable for the clothing pressure test.

Since the output signal of Flexiforce sensor is quite weak, amplifying circuit is engaged to facilitate the conversion and data processing. According to engineering design, pressure detection of eight measurement points should be detected at the same time. Therefore, two Quad- Operational -Amplifier MC34074 are employed in the proposed detection system to measure four point pressure signals respectively.

NI SCXI is a high performance signal conditioning and switching platform for measurement and automation system, and its data acquisition (DAQ) hardware system is composed of multi-channel signal conditioning modules. Flexiforce sensor will convert pressure to a voltage signal, and this analog signal is amplified by MC34074 amplifier amplification circuit. The output signal of MC34071 is then given to the SCXI A / D conversion module for converting to a digital signal, which is eventually sent to host computer to realize the pressure real-time monitoring and data processing.

\section{Software Design of Detection System}

Calibration of Detection System. In order to determine the linearity of the detection system, the static calibration of the system circuit is required ${ }^{[4]}$. The method of calibration is to place the standard weights directly in the sensor area. Sensor then produces a voltage value because of the weight loading. After that, the pressure output values are measured and recorded, then a Pressure-Voltage curve diagram is drawn and the experience formula is obtained.

Usually we may assume that the relationship is a series of undetermined polynomials whose coefficients can be determined by computing. The method of fitting the data with empirical formula is also called regression analysis. Similarly, it can be called regression analysis if its empirical formula is linear, for example:

$$
y=c_{0}+c_{1} x_{1}+\cdots+c_{n} x_{n}
$$

This system independent variable is the pressure $\mathrm{N}$, and the actual test value is voltage $\mathrm{U}$, so the equation is linear regression model, that is linear fitting equation. Assuming the form of the fitting equation is:

$$
U=C_{0}+C_{1} N
$$

If there are actual measurement data $\left(N_{i}, U_{i}\right), \quad i=1,2,3 \cdots, n$, whose any independent variable $N_{i}$ corresponds to the estimated values $\widehat{U}_{i}$ in the fitting line, the error between the estimated value and the actual test value can be computed as $e_{i}=U_{i}-\widehat{U_{i}}$. According to the principle of least squares fitting, the square of residual error should be minimum, that is, the $\sum_{i=1}^{n} e_{i}^{2}$ is minimum. Therefore, calculate the $\sum_{i=1}^{n} e_{i}^{2}$ first order partial derivatives for $\mathrm{C}_{0}$ and $\mathrm{C}_{1}$ respectively, and make the formula equal to zero: 


$$
\begin{aligned}
& \frac{\partial \sum_{i=1}^{n} e_{i}}{\partial C_{0}}=0 \\
& \frac{\partial \sum_{i=1}^{n} e_{i}}{\partial C_{1}}=0
\end{aligned}
$$

Bring practical measured data into the formula (3), (4) respectively, and obtain values $\mathrm{C}_{0}, \mathrm{C}_{1}$ by computing; Substitute $\mathrm{C}_{0}$ and $\mathrm{C}_{1}$ into formula (2), and further conversion to:

$$
N=\left(\frac{U-C_{0}}{C_{1}}\right)
$$

The formula (5) is applied to the design of the LabVIEW program, which can be used in the actual engineering to realize the conversion of voltage to pressure.

Fitting Program Design. In order to acquire 8 pressure signals, it is necessary to carry out the static calibration of the 8 pressure sensors respectively. First, by using the method of direct loading Weight, each voltage value will be collected by the data acquisition program, and then the experimental data are sent to the fitting program designed for fitting processing. The method of fitting procedure for each signal is the same, and one of the fitting procedures is shown in Figure 2 . The collected experimental data are filled in the sample array of front end, and this subroutine will fit a curve, and calculate the coefficient and fitting equation and the variance of the MSE. The smaller square error (MSE) indicates the smaller fitting error and better fitting effectivity ${ }^{[5]}$.

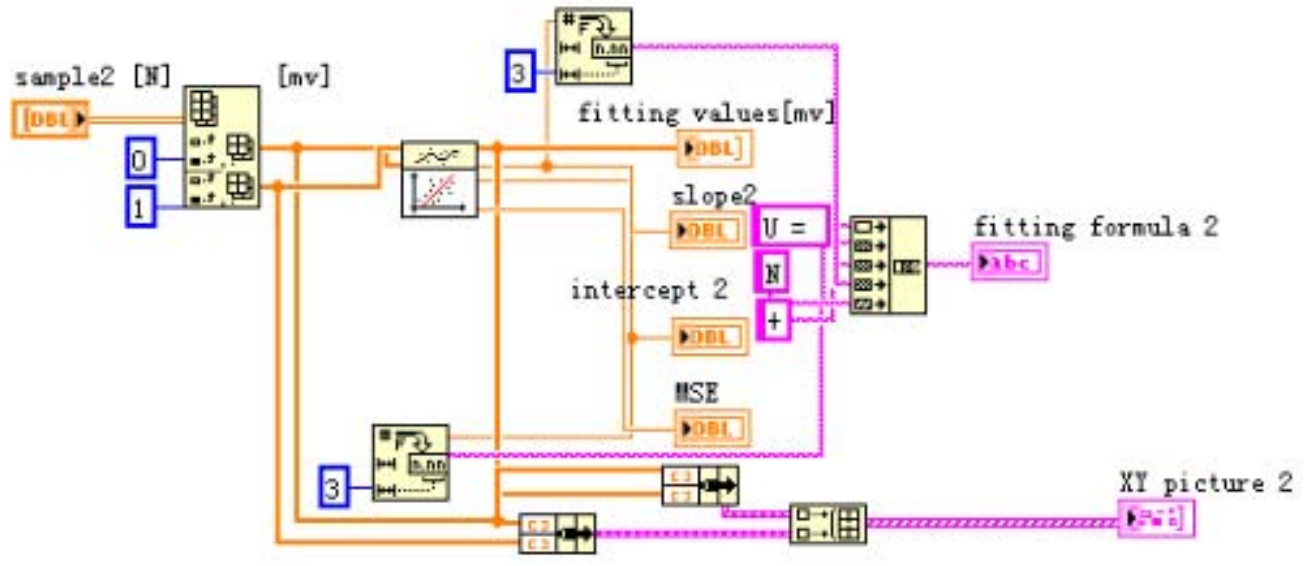

Fig. 2 Least square fitting program design

Bring the parameters $\mathrm{C}_{0}$ (intercept) and $\mathrm{C}_{1}$ (slope) calculated by fitting procedure into transformation formula (5), then the voltage value will be converted to pressure value through LabVIEW local variables. The specific program block diagram is shown in Fig. 3.

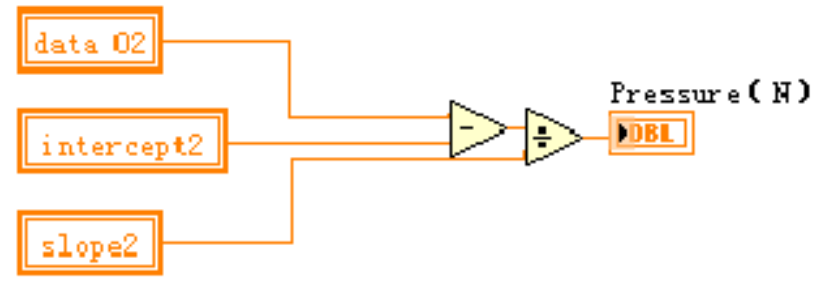

Fig. 3 Voltage conversion to pressure flow chart 


\section{System Running Test}

In order to verify the effect of the least squares fitting, objects of different weights are placed in the Flexiforce sensor area. Then, running this pressure detection system and the results are obtained as shown in Fig. 4.

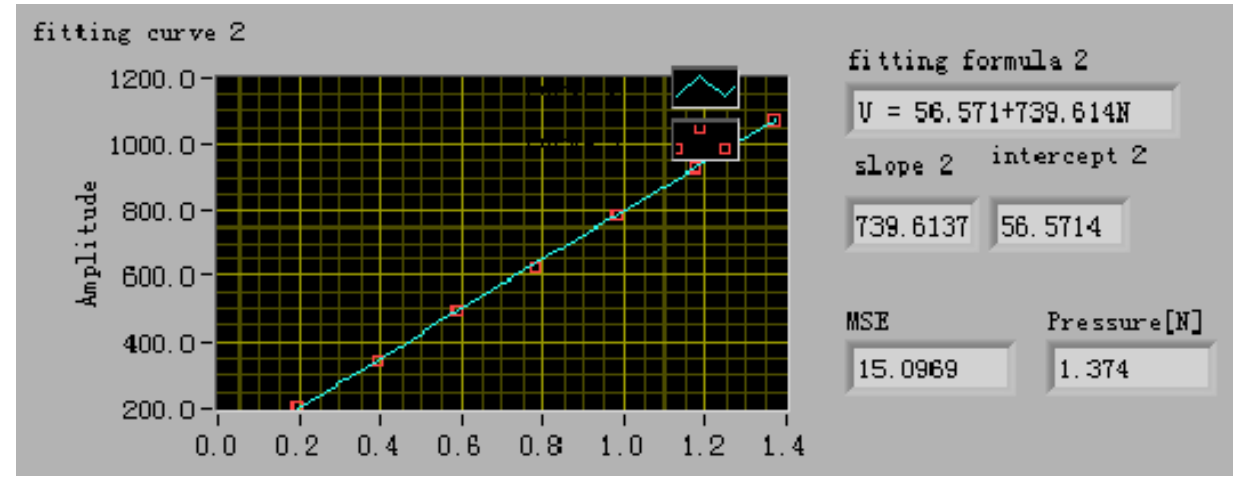

Fig. 4 System running test result

The results can be seen from the results of the fitting of the data is linear distribution. Set the second pressure sensor experimental data as an example. The mean square value say, MSE, is 15.0969. Table1 shows the comparison of fitting value obtained by least square fitting algorithm and the actual measurement values. It is obviously found the error between the fitting value and the actual measurement value is less than $9.5 \mathrm{mV}$. This error could meet the engineering needs basically.

Table 1 The comparison between fitting values and measured values

\begin{tabular}{|c|c|c|c|c|c|c|c|}
\hline Number & 1 & 2 & 3 & 4 & 5 & 6 & 7 \\
\hline measured values [mv] & 204 & 347 & 493 & 627 & 783 & 928 & 1073 \\
\hline fitting values [mv] & 201.53 & 346.5 & 491.464 & 636.429 & 781.393 & 926.357 & 1071.32 \\
\hline
\end{tabular}

\section{Conclusion}

In this paper, a pressure detecting system is developed for clothing pressure testing. Based on LabVIEW software platform, this system calibrates the acquired pressure data with least square fitting algorithm. It achieves pressure real-time data analysis and dynamic objective test. Test running shows the reliability and effectivity of the proposed pressure detection system. The possible research direction may include data filtering, error analysis and compensation algorithm design.

\section{Acknowledgements}

This study was financially supported by National Natural Science Funds of China (Grant No. 51206021) and Shanghai Committee of Science and Technology, China (Grant No. 12ZR1400700).

\section{References}

[1] Chen Hongjuan and LiWei: A research on the testing of the garment pressure on body. Knitting Industry.(2006), p. 53

[2] Wu Shunqiu: The Study of General LS Linear Fit Based on LabVIEWB 8.5. Journal of Yangtze University (Nat Sci Edit). (2009), p. 119

[3] Zhou Runjing, Hao Xiaoxia, in: Sensor and Detection Technology, edited by Zhang Jian, Publishing House of Electronics Industry, Beijing (2009). 
[4] Meng Zhenhua, Lijin: A Study on a New Test Ways of Clothing Pressure. Shandong Textile Science and technology.(2006), p. 39

[5] Huang Shengmin, Tianye: A design of the instrument of Linear fit on LabVIEW. Natural Sciences Journal of Harbin Normal Univessity.(2006), p. 61 